\title{
Predictive Model For Preliminary Assessment of Repeated Radioiodine Therapy Course Based On 131-I Suv
}

\section{Alexandr A Bubnov ( $\sim$ bubnov96@mail.ru )}

Center of Endocrinological Research: Centro de Investigaciones Endocrinologicas

https://orcid.org/0000-0002-5877-6982

\section{Alexey A Trukhin}

Endokrinologicheskii nauchnyi tsentr

\section{Pavel O Rumyantsev}

St Petersburg Sogaz

\section{Mikhail V Degtyarev}

Endokrinologicheskii nauchnyi tsentr

\section{Yaroslaw I Sirota}

Endokrinologicheskii nauchnyi tsentr

\section{Sergey S Serzhenko}

Endokrinologicheskii nauchnyi tsentr

\section{Marina S Sheremeta}

Endokrinologicheskii nauchnyi tsentr

Konstantin Yu Slashchuk

Endokrinologicheskii nauchnyi tsentr

\section{Natalya G Mokrysheva}

Center of Endocrinological Research: Centro de Investigaciones Endocrinologicas

\section{Original research}

Keywords: Standardized Uptake Value (SUV), SPECT/CT, radioiodine therapy, differentiated thyroid cancer, image quantification

Posted Date: July 22nd, 2021

DOl: https://doi.org/10.21203/rs.3.rs-613524/v1

License: (c) (1) This work is licensed under a Creative Commons Attribution 4.0 International License.

Read Full License 


\title{
PREDICTIVE MODEL FOR PRELIMINARY ASSESSMENT OF REPEATED RADIOIODINE THERAPY COURSE BASED ON 131-I SUV
}

Author Block: Alexandr A. Bubnov, Alexey A. Trukhin, Pavel O. Rumiantsev., Mikhail V. Degtyarev., Yaroslaw I. Sirota, Sergey S. Serzhenko, Marina S.Sheremeta, Konstantin Yu Slashchuk. Natalya G. Mokrysheva

\section{Author information}

The National Medical Research Center for Endocrinology. Institute of Oncoendocrinology, Department of radionuclide therapy, Dmitry Ulyanov street 11,Moscow, Russia

Alexandr A. Bubnov ORCID: https://orcid.org/0000-0002-5877-6982

Alexey A. Trukhin ORCID: https://orcid.org/0000-0001-5592-4727

Marina S.Sheremeta. ORCID: https://orcid.org/0000-0003-3785-0335

The National Medical Research Center for Endocrinology. Institute of Oncoendocrinology, Department of radionuclide diagnosis,Dmitry Ulyanov street 11, Moscow, Russia

Mikhail V. Degtyarev ORCID: https://orcid.org/0000-0001-5652-2607

Yaroslaw I. Sirota ORCID: https://orcid.org/0000-0002-0613-9543

Sergey S. Serzhenko ORCID: https://orcid.org/0000-0003-2326-1396

Konstantin Yu Slashchuk ORCID: https://orcid.org/0000-0002-3220-2438

The National Medical Research Center for Endocrinology. Dmitry Ulyanov street 11, Moscow, Russia

Natalya G. Mokrysheva ORCID: https://orcid.org/0000-0002-9717-9742

"IMC SOGAZ” St. Petersburg, Russia

Pavel O. Rumiantsev ORCID: https://orcid.org/0000-0002-7721-634X

Corresponding author

Correspondence to Alexandr A. Bubnov email: bubnov96@mail.ru

\begin{abstract}
Purposes: Current research design is dedicated to 131-I SUV evaluation on post therapeutic scintigrams and setting up predictive model for radioiodine therapy repeated course prescription in differentiated thyroid cancer (DTC). Methods: Study includes 148 patients (f-105, m-43) with DTC treated with 131-iodine. Administered treatment activities of 131I calculated according to clinical features and tumor recurrence risk group. Patients were divided into three groups using ATA 2018 recommendations. Absolute risk groups: low risk (L), medium risk $(\mathrm{M})$, high risk $(\mathrm{H})$. Administered 131-I activity $[\mathrm{MBq}]:\langle\mathrm{AL}\rangle=3223 \pm 729,\langle\mathrm{AM}\rangle=3696 \pm 456,\langle\mathrm{AH}\rangle=$ 4589 $\pm 1078 ; \mathrm{Tg}[\mathrm{ng} / \mathrm{ml}]:\langle\mathrm{TgL}\rangle=7,4 \pm 1.7 \mathrm{ng} / \mathrm{ml},\langle\mathrm{TgM}\rangle=14,8 \pm 5,9,\langle\mathrm{TgH}\rangle=68,3 \pm 18,5 ; \mathrm{TgAb}[\mathrm{IU} / \mathrm{ml}]:$
\end{abstract}


$\langle\mathrm{TgAbL}\rangle=124,3 \pm 81.7,\langle\mathrm{TgAbM}\rangle=29,2 \pm 15,9,\langle\mathrm{TgAbH}\rangle=85,7 \pm 28,9$. All null hypothesis were checked using paired Mann-Whitney U-test. Calibration of system SPECT/CT and evaluation SUVs completed according to protocols designed using Jaszczak phantom Deluxe. Model development based on logistic regression with ROC-analysis, regularization and cross-validation. Results: Reference intervals of SUVpeak and SUVmax calculated for all groups of risk. SUVpeak: low risk $>155$, medium risk 105-155, high risk 0-105 pL-M=0.069 pL-H=0.0037 pM-H=0.7514; SUVmax: low risk >38, medium risk 29-38, high risk 0-29 pL-M=0.052 pL$\mathrm{H}=0.0033$ pM-H=0.949. Logit model based on SUVpeak without regularization has: AUC $=0.67$ (95\%CI 0.331.00); Accuracy $=0.82 ; \mathrm{SE}=0.89 ; \mathrm{SP}=0.4 ; \mathrm{PPV}=0.41 ; \mathrm{NPV}=0.89$, cross-validated AUC $=0.67(0.4-0.88)$, regression coefficients: B0=0.037, B1=0.001. Regularization (SUVpeak $<100)$ lead to AUC $=0.75$, (95\%CI 0.44$1.00)$; accuracy $=0.89 ; \mathrm{SE}=0.98 ; \mathrm{SP}=0.4 ; \mathrm{PPV}=0.76 ; \mathrm{NPV}=0.87$, cross-validated $\mathrm{AUC}=0.513(0.36-0.71)$ regression coefficients: $\mathrm{B} 0=0.473, \mathrm{~B} 1=0.003$.

Conclusion: Study shows that SUV has wide range of values and can be matched with existed model of risk assessment of DTC. Algorithm of image segmentation and evaluation of SUVpeak and SUVmax for SPECT/CT systems was developed. According to the ROC analysis, developed predictive model shows an acceptable performance for further clinical investigation and advancement focused on refining model parameters and introducing additional predictors

\section{Keywords}

Standardized Uptake Value (SUV), SPECT/CT, radioiodine therapy, differentiated thyroid cancer, image quantification

\section{Ethics approval and consent to participate}

This article contains studies with human participants performed by authors. Before that, written consent was obtained from all patients

\section{Consent for publication}

All authors gave consent for publication

\section{Availability of data and material}

All data generated or analysed during this study are included in this published article (Method for assessing the risk of recurrent differentiated thyroid cancer after radioiodine therapy. Bubnov AA, Trukhin AA, Rumiantsev PO, Degtiarev MV, Serzhenko SS, Slashchuk KYU, Kolpakova EA, Dedov II, Mokrysheva NG, Melnichenko GA. ru) 2743275 C1. Patent Office: Russia. Year of publication: 2021 Application number: 2020128431 Date of registration: 26.08.2020 Date of publication: 16.02.2021).

\section{Competing interests}

The authors declare no conflict of interest

Funding The study was supported by Ministry of Science and Higher Education of the Russian Federation (agreement no. 075-15-2020-899)

\section{Authors' contributions}

Bubnov A.A. - study conception and design, implementation of all research stages, create predictive model, data analysis, illustrations, drafting of the manuscript, critical review for important intellectual content. 
Rumiantsev P.O. - study conception and design, data analysis, illustrations, drafting of the manuscript, critical review for important intellectual content.

Trukhin A.A. - study conception and design, implementation of all research stages, create predictive model data analysis and interpretation, critical review for important intellectual content.

Degtyarev M.V. - post-therapy image analysis, critical review for important intellectual content

Sirota Y.I. - drafting of the manuscript,post-therapeutic image processing.

Serzhenko S.S. - post-therapy image analysis,illustrations

Sheremeta M.S. - critical review for important intellectual content

Slashchuk K.Y. - stratification for the risk of absolute relapse, drafting of the manuscript

Mokrysheva N.G. - critical review for important intellectual content

\section{Acknowledgements}

We thank the Ministry of Science and Higher Education of the Russian Federation and The National Medical Research Center for Endocrinology for help to realize the study

\section{Introduction}

People have faced different diseases throughout human history and seek to find effective methods for solving the problems. Currently, medicine is quickly developing. It combines technologies and achievements of fundamental scientific disciplines such as medicine, physics, chemistry and biology. Interdisciplinary approach to problem increases the diagnostic efficiency for revealing diseases at an early stage significantly.

Standardized Uptake Value (SUV) of radiopharmaceuticals (RPs) is one of the most perspective group of parameters in nuclear medicine [1]. The SUVs are feature of quantitative analysis with application of hybrid SPECT/CT systems, that have proved its usefulness in PET/CT but absence of it's application in SPECT/CT set an ambitious task for evidence-based instrumental diagnostics [2, 3]. The departments change their own approach to treatment patients from classical such as evaluation of current uptake value (counts in region of interest (ROI) divided by counts in whole body mode on scintigraphy image) and description of ROI, to quantitative - radiomics.

Radiomics is based on creating a complex of digital patient parameters on image different modality [4]. SUV is a part of radiomics and describes tissue pharmacokinetics in current state. For assessing the value investigators conduct phantom studies and design a sequence of measurements [5]. One of the main advantages of SPECT/CT visualization is a lot of medical radionuclide compared with PET/CT. It gives an opportunity to expand the scope of quantitative assessment metabolic activity in addition to the PET facilities [6,7].

Quantitative description of metabolic activity tissue is an important part of treatment. It allows to define therapeutic activity for achievement of treatment effect from radionuclide therapy. In addition, the approach to processing images gives the opportunity to clarify risk of absolute relapse. Consequently, doctors can make predictions about the number of repeated radionuclide therapy courses [8]. 
Annual increase of new endocrine disease cases equals $4.5 \%$ [9]. Among endocrine system diseases, the leading position has pathologies of thyroid gland (malignant and benign neoplasms) [10]. Therefore, there is a need to improve the quality of diagnostics. Processing images transform from description level to quantitative, which includes development and implementation method for measurement of metabolic activity thyroid tissue and metastasis process using SPECT/CT system. There are countries such as South Korea, the USA, Germany and Japan that actively implement SUVs in medical protocols [11-13].

Current research design is dedicated to 131-I SUV evaluation on post therapeutic scintigrams, receiving references range of SUVpeak and SUVmax depending on absolute risk of relapse in differentiated thyroid cancer (DTC). Predictive model for appointment the second course of radioiodine therapy (RIT) in DTC was created to improve effective outcome and acceptable safety.

\section{Material and methods}

Patient inclusion

Present study included 250 patients with diagnosed DTC and after radioiodine therapy was done in the nuclear medicine department (Endocrinology Research Center) from January to December in 2018. There were 198 patients with confirmed histology and 148 of them had information about level Tg and AT-Tg. Children were excluded from the research. After thyroidectomy patients had euthyroid status or obtained suppressive therapy with levothyroxine. Prescribed activities of 131I calculation were based on protocol of medical profile. Patient cohort was divided into three groups according to (ATA 2018) TNM risk assessment [14].

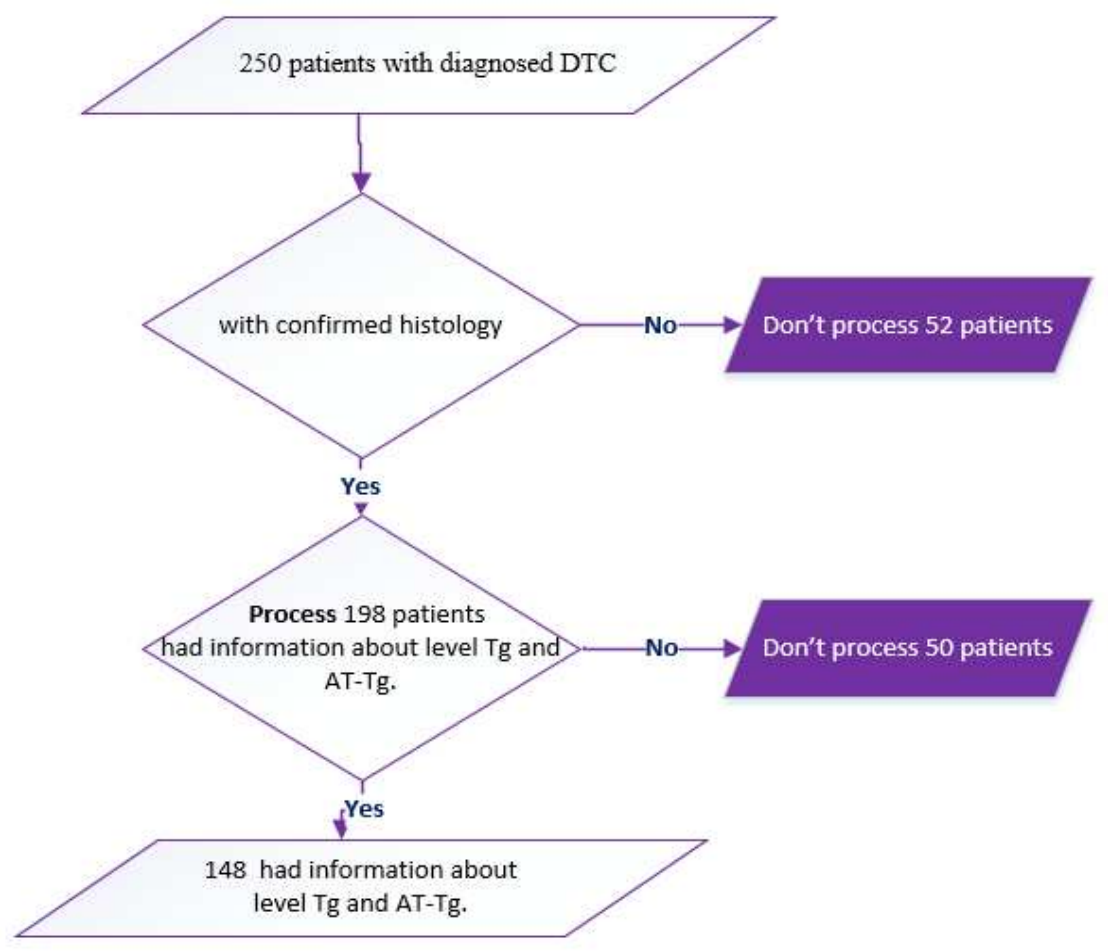

Fig. 1. Selection of patients for further processing 


\section{Calibration protocol of SPECT/CT system GE Discovery NM/CT 670 with using phantom Deluxe Jasjczak (model ECT/DLX/P)}

Manual for fused SPECT/CT examinations were developed for SPECT/CT GE DISCOVERY NM/CT 670. According to the protocol some parameters of SPECT/CT system was obtained such as sensitivity, spatial resolution (FWHM), dependence spatial resolution from depth of radiation source position and influence of the dead time of the spectrometric path.

SPECT/CT GE Discovery NM/CT 670 was calibrated using Deluxe Jasjczak (Model ECT/DLX/ P) (Fig. 2). SPECT/CT configuration: HEGP collimator; exposition time - $600 \mathrm{sec} ; 364 \mathrm{KeV}$ energy window $-20 \%$ with scatter correction window - 10\%; $\mathrm{U}=120 \mathrm{KV}, \mathrm{I}=400 \mathrm{~mA}$ - x-ray tube; image matrix - 128x128; thickness of CTslice - $1.25 \mathrm{~mm}$. Image processing software - GE Medical Systems Xeleris 4.0 (Volumetrix MI). Reconstruction parameters: reconstruction method-OSEM, number of iterations -5 ; subset - 10, filter - Butterworth; attenuation correction, scatter correction, resolution recovery were applied.

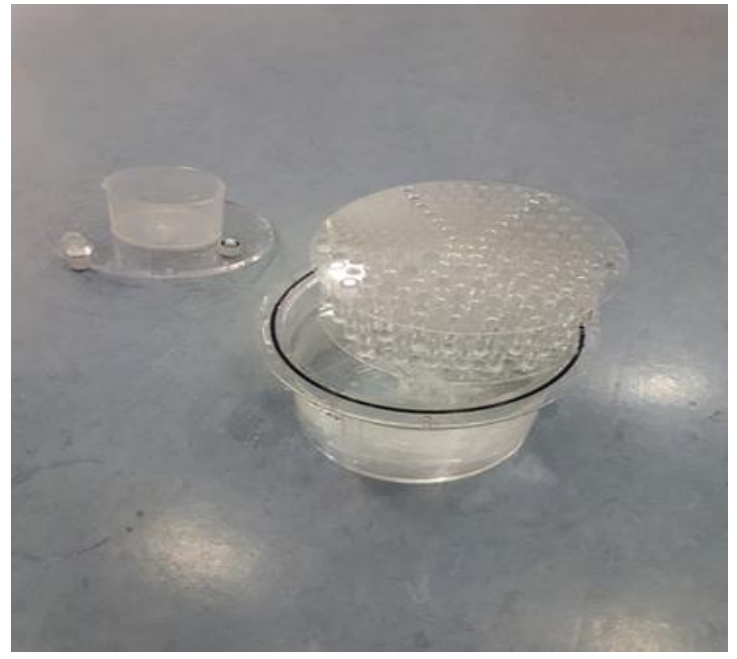

Fig.2. Phantom Deluxe Jasjczak (model ECT/DLX/P).

\section{Sensitivity test of SPECT/CT system}

The camera calibration factor (sensitivity) $\mathrm{S}$ is determined according to

$$
\mathrm{S}=\frac{\dot{\mathrm{C}}_{\mathrm{VOI}}}{\mathrm{A}_{\mathrm{VOI}}}(1)
$$

$\dot{C}_{V O I}$ is the count rate measured in the VOI, and Avoi is the decay-corrected activity in the VOI. Deluxe Jasjczak (Model ECT/DLX/ P) was completely filled with water (without air bubbles). Furthermore, activity of 131-I was injected in the volume of phantom (activity in syringe A=248 MBq) (Fig 3.). 


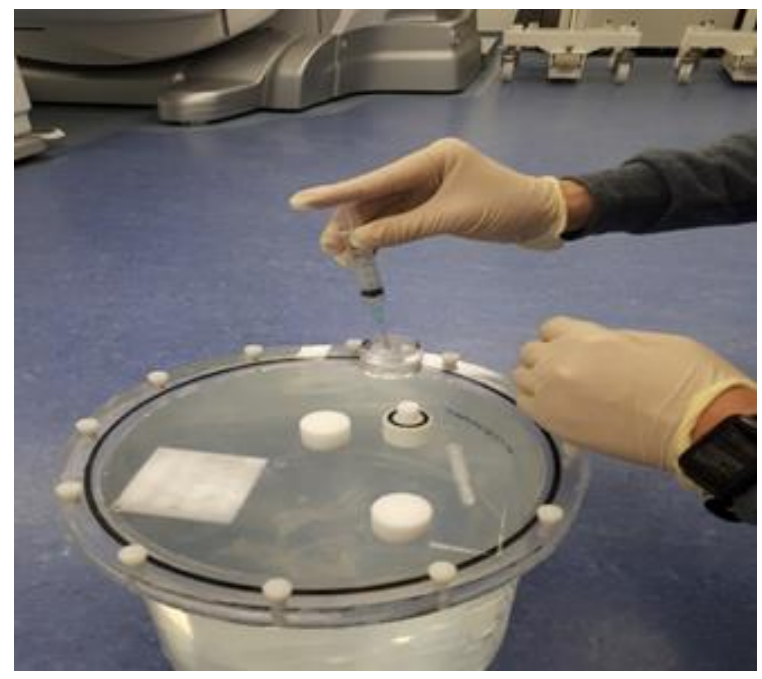

Fig 3. Administration 131-I into phantom volume.

After that, for equal distribution of activity in Deluxe Jasjczak was shaken. At the beginning of scanning phantom was positioned in the central part on the table of SPECT/CT system. Furthermore, SPECT/CT was checked by automatic mode. At the same time, a protocol of scan was loaded and the study record was created. Finally, information about characteristics of radiation source was combined using SPECT/CT system (the first part of scan was emission and the second was CT-part) (Fig 4)

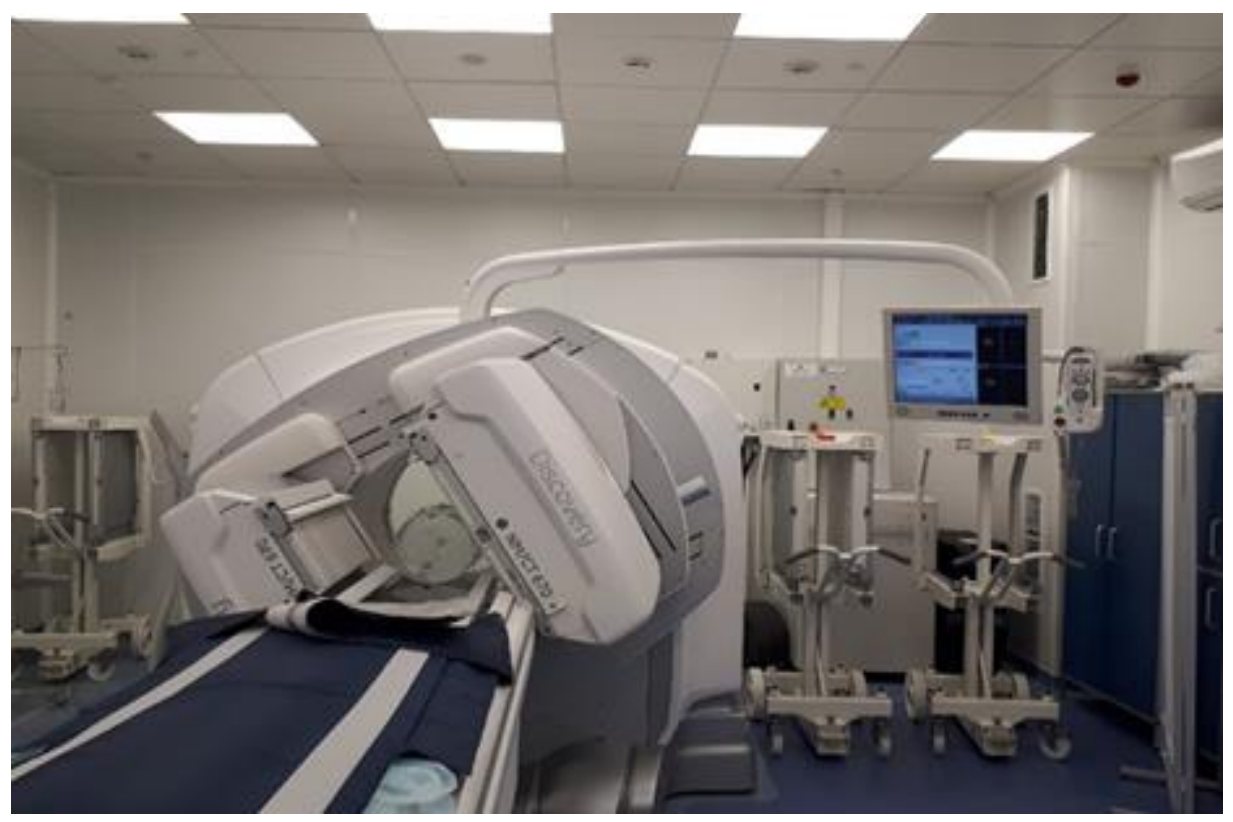

Fig 4. Scanning of phantom

During the study, six series of scans were accomplished. Before each series was fixed time of activity administration, value of activity in phantom and remaining activity in syringe measuring a dosage calibrator. Eventually, the results of each scans were transferred to Xeleris workstation. There was Volumetrix MI (GE Healthcare). The application is created for processing of tomography images where image reconstruction is performed. A reconstruction was done with certain parameters, which were considered at the top of the topic. The reconstructed image was processed using MATLAB. 
The research conducted with 131-I, but from further experiments it can be concluded that the results are identical for any radionuclides. Activity of radionuclide in phantom during the study was 22,3 $\mathrm{MBq}$ with correction of radiation decay. Average count with image and slice sensitivity were studied during processing the reconstructed images.

\section{FWHM study depending on the source positioning depth}

In order to determine FWHM of SPECT/CT (emission part) insulin syringes with 131-iodine (model of point source) were put in phantom volume in distinct depth in a special cap (Fig 5).

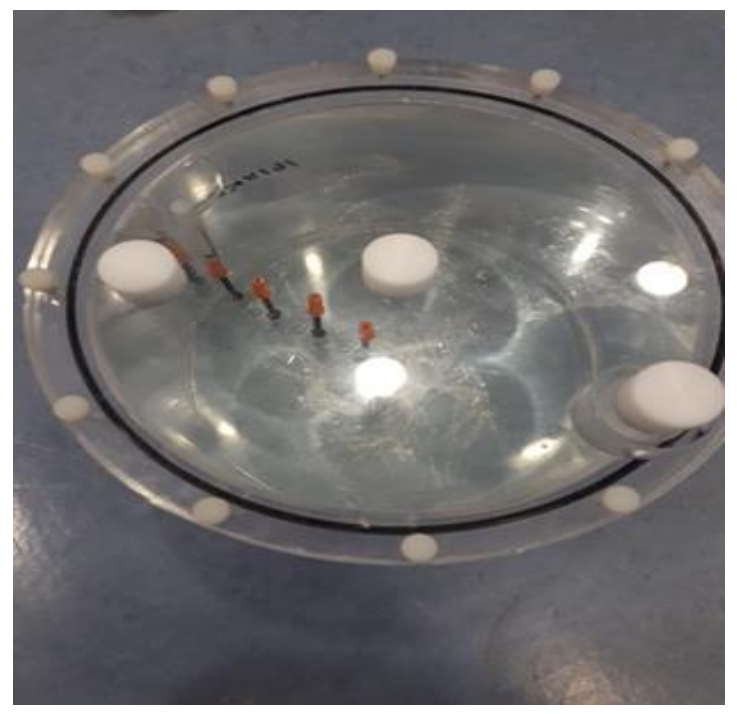

Fig 5. Positions of insulin syringes with 131-iodine in the phantom volume

Radioactive liquid volume of insulin syringe was $0,09 \mathrm{ml}$. At the beginning of the study iodine activity was $1,7 \mathrm{MBq}$. The steps of planning and scanning were carried out similarly to the sensitivity test. The syringe positioning depth was determined by CT (Fig 6). The first series of measurements was implemented at a depth of $0.73 \mathrm{~cm}$.

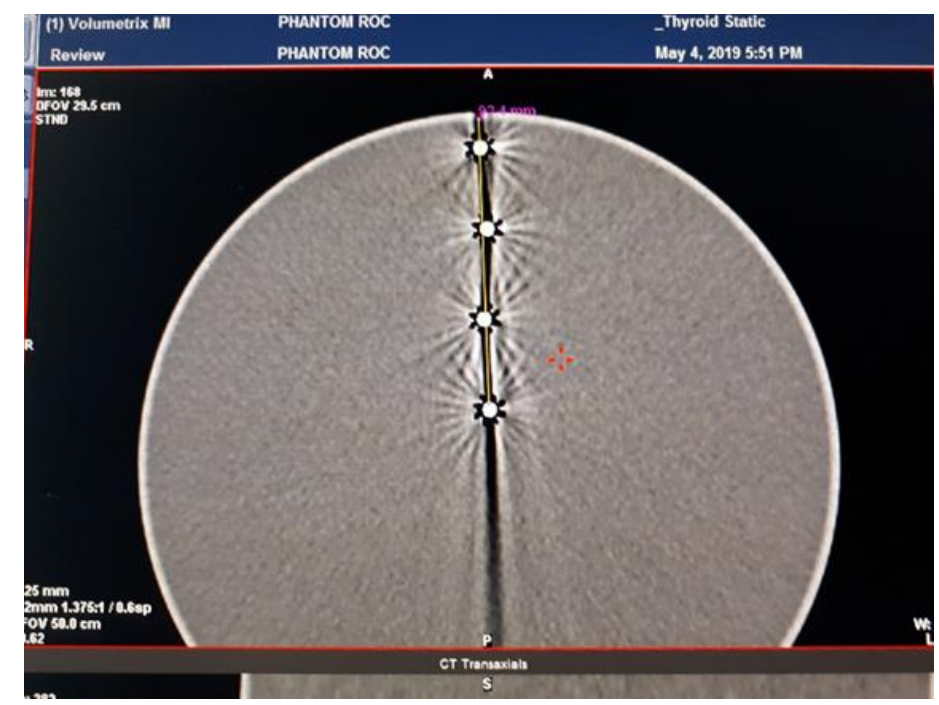


Fig 6. Measuring positioning depth of the syringe with activity by CT.

The syringe was as close as possible to the side wall of the phantom. The last time insulin syringe was put at a depth of $9.24 \mathrm{~cm}$ (Fig 7).

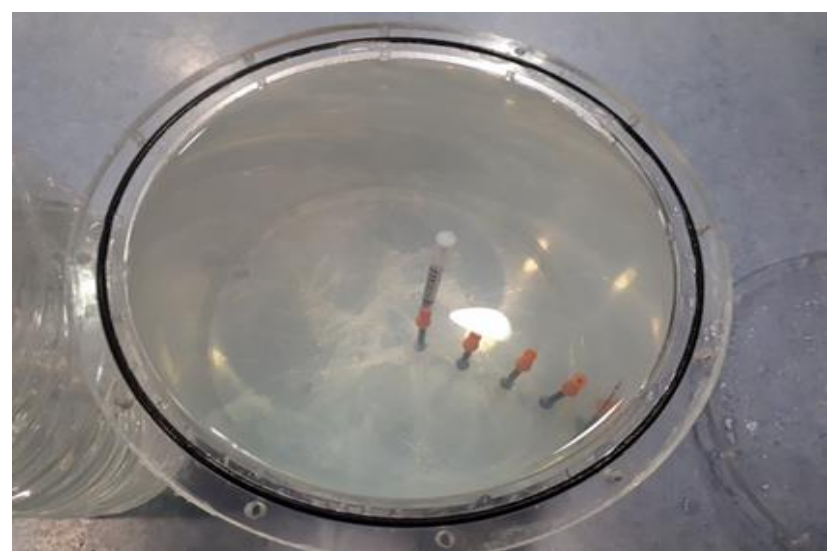

Fig 7. The last position of insulin syringes with 131-iodine in the phantom volume

Each study at the appropriate depth consisted of three sequential scans. The first one included SPECT/CT system and following scans contained only emission part. Eventually, the results of each scan were transferred to Xeleris workstation. After that, MATLAB was used for visualization of the distribution of the count over the slices. As a result FWHM was obtained in slice units. FWHM in slice units multiplied by the slice thickness in $\mathrm{mm}$.

\section{Method for determining the group of parameters SUVs (SUVpeak, SUVmax, SUVmean)}

Input data for evaluation SUV: Therapeutic activity of RPs at the time of scanning(MBq); Total research score (count); Study duration (sec); Number of projection (pieces); Time per projection (sec); Sensitivity of SPECT/CT system (count/MBq*sec); Maximum count in ROI (count); Total count in ROI (count); Volume of segmentation area $(\mathrm{ml})$; Patient body weight $(\mathrm{kg})$; density of ROI tissue $(\mathrm{kg} / \mathrm{m} 3)$.

Therapeutic activity of RPs was determined 72 hours after administration with correction for decay. After that, scanning of patient was carried out using SPECT/CT system. Subsequently, information about the research was picked up: total research score, total count in sphere $1 \mathrm{ml}$ or total count in ellipsoid with linear size getting with CT, maximum count in voxel, volume of ellipsoid with linear size getting with CT or volume sphere $1 \mathrm{ml}$. Total research score was inserted in dependence of the tomography sensitivity from projection count rate.

The sensitivity value was substituted in formula to find activity in ROI, respectively:

$$
\mathrm{A}=\frac{C_{\text {ount }}}{s \cdot t}(2)
$$

$\mathrm{S}$ - sensitivity (this value from phantom study. count/MBq*sec), Count - Total count in ROI after segmentation it, $\mathrm{t}$ - time of exposition ( $\mathrm{sec}$ ).

Three type of activity were calculated during the study: 


$$
\text { Amean }=\frac{\operatorname{Count}(\text { mean })}{S \cdot t} \quad \text { Apeak }=\frac{\operatorname{Count}(\text { peak })}{S \cdot t} \quad \text { Amax }=\frac{\operatorname{Count}(\text { max })}{S \cdot t}
$$

(a) (b) (c)

(a)Amean - activity in focus (MBq), (b) Apeak - activity in sphere $1 \mathrm{ml}(\mathrm{MBq})$, (c)Amax - maximum activity $(\mathrm{MBq})$. Count(mean) - total count in ROI, Count(peak) - count in sphere $1 \mathrm{ml}$, Count(max) - maximum count in $\operatorname{voxel}(0,086 \mathrm{ml})$.

SUVs were calculated by the following formulas:

$$
S U V_{\text {meam }}=\frac{\frac{A_{\text {mean }}}{\rho \cdot V}}{\frac{A_{\text {inj }}}{m}} \quad S U V_{\text {peak }}=\frac{\frac{A_{\text {peak }}}{\rho \cdot V_{\text {peak }}}}{\frac{A_{\text {inj }}}{m}} \quad S U V_{\text {max }}=\frac{\frac{A_{\text {max }}}{\rho \cdot V_{\text {vox }}}}{\frac{A_{\text {inj }}}{m}}
$$

(a) (b) (C)

(a) SUVmean - mean SUV in ROI, (б) SUVpeak - SUV in sphere $1 \mathrm{ml}$, (в) SUVmax - SUV in voxel with maximum count. Amean - activity in focus (MBq), Apeak - activity in sphere $1 \mathrm{ml}(\mathrm{MBq})$, Amax - maximum activity (MBq), $\rho$ - density of thyroid tissue (g/cm3), V - volume ROI (ml), Vpeak - volume sphere 1 ml, Vvox volume 1 voxel $(0,086 \mathrm{ml}), \mathrm{m}$ - mass of patient body. Ainj - injected activity (MBq).

\section{Statistical analysis}

In this research, the GraphPad Prism 8.0 package was used for statistical analysis. The instrument was used for processing and interpretation results of the investigation. Also it was a foundation for formation of SUVs reference intervals. At first, for each cohort was done the normal test and for all parameters. Normal test was failed for all cohorts because of it the data were processed like nonparametric methods. Also, according to all available evidence for statistical analysis was chosen non-parametric Mann-Whitney test.

\section{Logistic regression}

Logistic regression is used for predicting the likelihood of some event, when there is a binary result yes/no disease [15]. In contrast to linear regression logistic regression doesn't predict numeric variable values from a sample of initial values. Instead of it, value of function is a probability that initial value belongs to a certain class. Logistic regression equation:

$$
\operatorname{logit}(p)=B_{0}+B_{1} \cdot x_{1}+B_{2} \cdot x_{2}+. .+B_{n} \cdot x_{n}
$$


$\mathrm{p}$ - probability that a patient with the predictor has positive results; $\mathrm{B} 0, ; \mathrm{x} 1, \mathrm{x} 2 \ldots \mathrm{xn}$ - corresponding predictor value. Method of maximum likelihood estimation is utilized to get logistic regression coefficients.After potentiating the formula 5

$$
\begin{aligned}
& \text { odds }=e^{\left(B_{0}+B_{1} \cdot x_{1}+B_{2} \cdot x_{2}+. .+B_{n} \cdot x_{n}\right)}=e^{t} \\
& B_{0}+B_{1} \cdot x_{1}+B_{2} \cdot x_{2}+. .+B_{n} \cdot x_{n} \text { stand } \mathrm{t}, \mathrm{t} \text { - function of chances. The ratio of chances }
\end{aligned}
$$

is

$$
\mathrm{P}=\frac{\text { odds }}{1+o d d s}
$$

After that the resulting P-value is compared with the selected threshold to conclude on true-positive cases.

\section{Creation of logistic regression model.}

At the beginning of creating a logistic regression model all patients were separated into several groups. Afterwards, training and test cohorts were set up. The model was based on 74 patients with high risk of absolute relapse according to ATA18 from the total cohort (148 patients). Among 74 patients was found 9 with proved the second course of radioiodine therapy. As a training group was distinguished cohort which was made up of patients with repeat (9 patients) and without it (11 patients). Test group contained 63 patients ( 9 with repeat and 54 without repeat). Logistic regression model was developed using MATLAB.

\section{ROC-analysis}

Cross-validation is a method for estimating work the model on a sample of independent data. ROCanalysis is a significant instrument, which allows to find parameters for getting the best and the worst results [16]. During the research was used following parameters:

- Sensitivity (Se) - percentage of correctly predicted repeated courses of RIT.

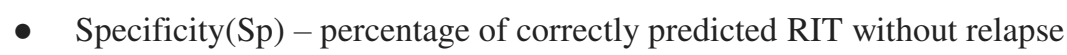

- Accuracy - percentage of correctly predictions relative to the total number of observations

- Positive Predictive Value (PPV) - percentage of patients with positive results of diagnosis test, which has relapse after the first course RIT

- Negative Predictive Value (NPV) - percentage of patients with negative results of diagnosis test, which doesn't have relapse after the first course RIT

Another indication of quality for the predictive model functioning is ROC (receiver operating characteristic)- curve. This method is based on a graphical representation of the relationship between sensitivity and specificity. It allows to analyze them and to solve an important problem linking with set a threshold for making diagnostic decisions at which the research errors will be minimal. In order to appreciate diagnostic ability of test is put up with a decision threshold. if P-value is higher than threshold than test will be positive and if P-value is lower than threshold than test will be negative. The most essential quantity that characterizes the ROC-curve is area under ROC-curve (AUC). AUC is determined according to 


$$
A U C=\int f(x) d x=\sum_{i}\left\lceil\frac{X_{i+1}+X_{i}}{2}\right\rceil \cdot\left(Y_{i+1}-Y_{i}\right)
$$

where $\mathrm{X}$ and $\mathrm{Y}$ - specificity and sensitivity, respectively.

\section{Results}

Dependence of the tomography sensitivity from count rate is linear (Fig 8). The graph shows that with increasing rate, the sensitivity decreases. Average value of sensitivity is $76 \pm 3 \mathrm{cps} / \mathrm{MBq} / \mathrm{sec}$.

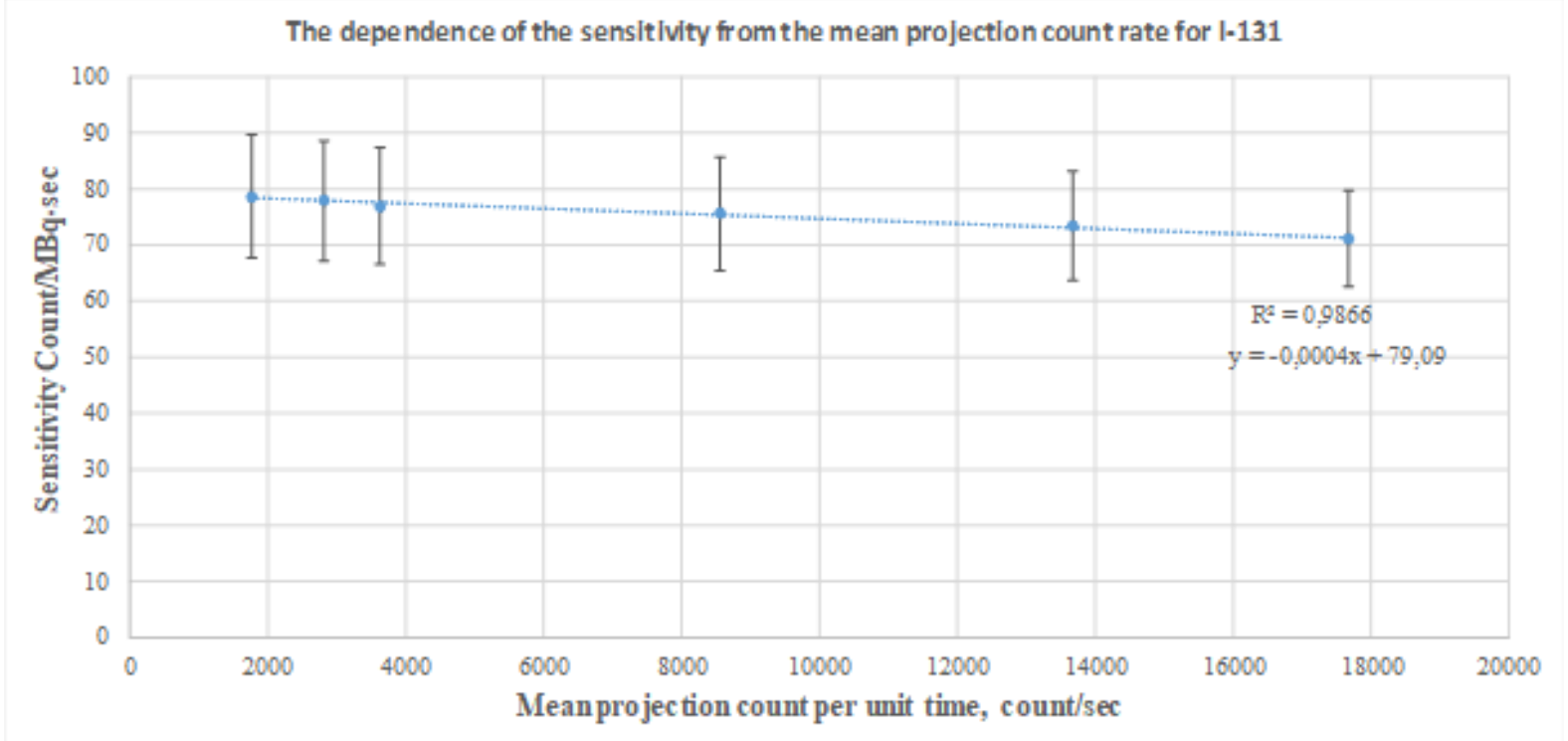

Fig. 8. The dependence of the sensitivity from the count rate in the phantom volume for iodine-131.

All scans were completed using Body Contour (device for po because sensitivity of SPECT is the same without Body Contour (device for positioning the gamma camera over the patient body) Therefore, Body Contour doesn't have affect on research results.

During the study the dependence of the sensitivity on the number of iterations was obtained (Fig 9). 


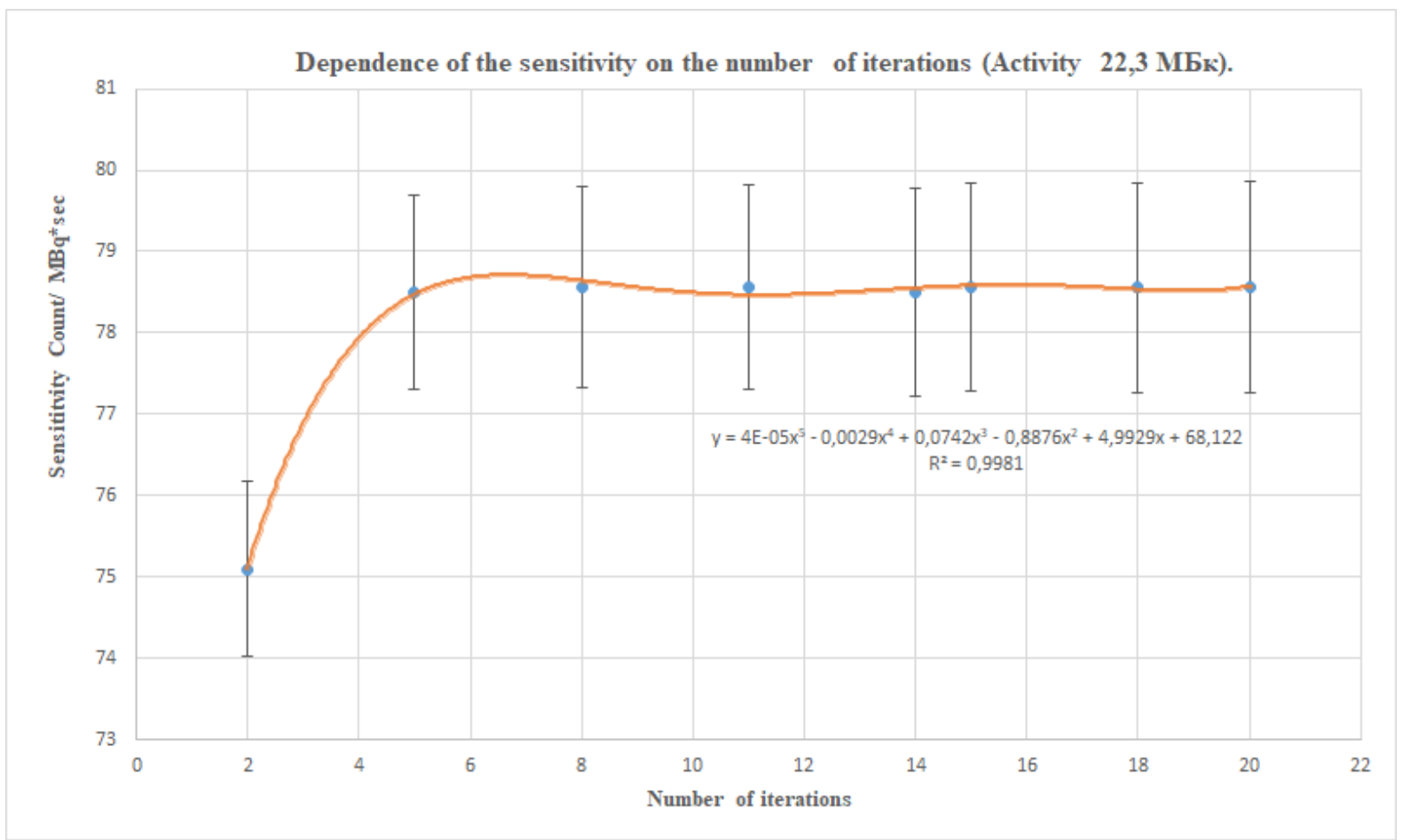

Fig. 9. Dependence of the sensitivity on the number of iterations.

Count value with increasing number of iterations was a gateway to the plateau and after 5 iterations was changed slightly. It allows to decrease processing time and minimize the influence of OSEM on reconstruction.

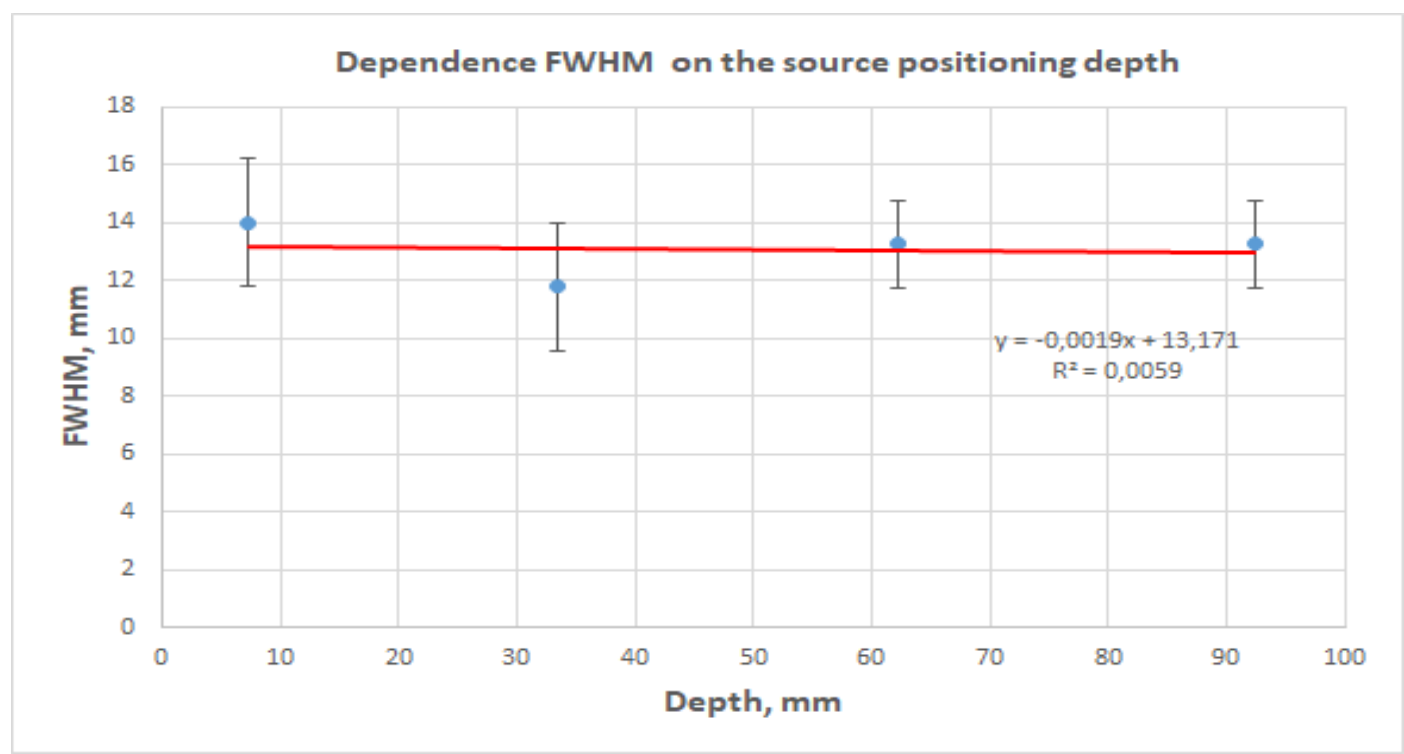

Fig. 10. Dependence FWHM on the source positioning depth

The investigation showed that behavior of FWHM changed slightly with depth (Fig 10). The deviation is observed in the area of depths more than $6 \mathrm{~cm}$. It is able to connect with edge effect on reconstruction images.

Radiologists' segmentation manual for fused SPECT/CT was developed during the study. Segmentation was carried out with helping software - GE Medical Systems Xeleris 4.0 (Volumetrix MI) (Fig 11). 


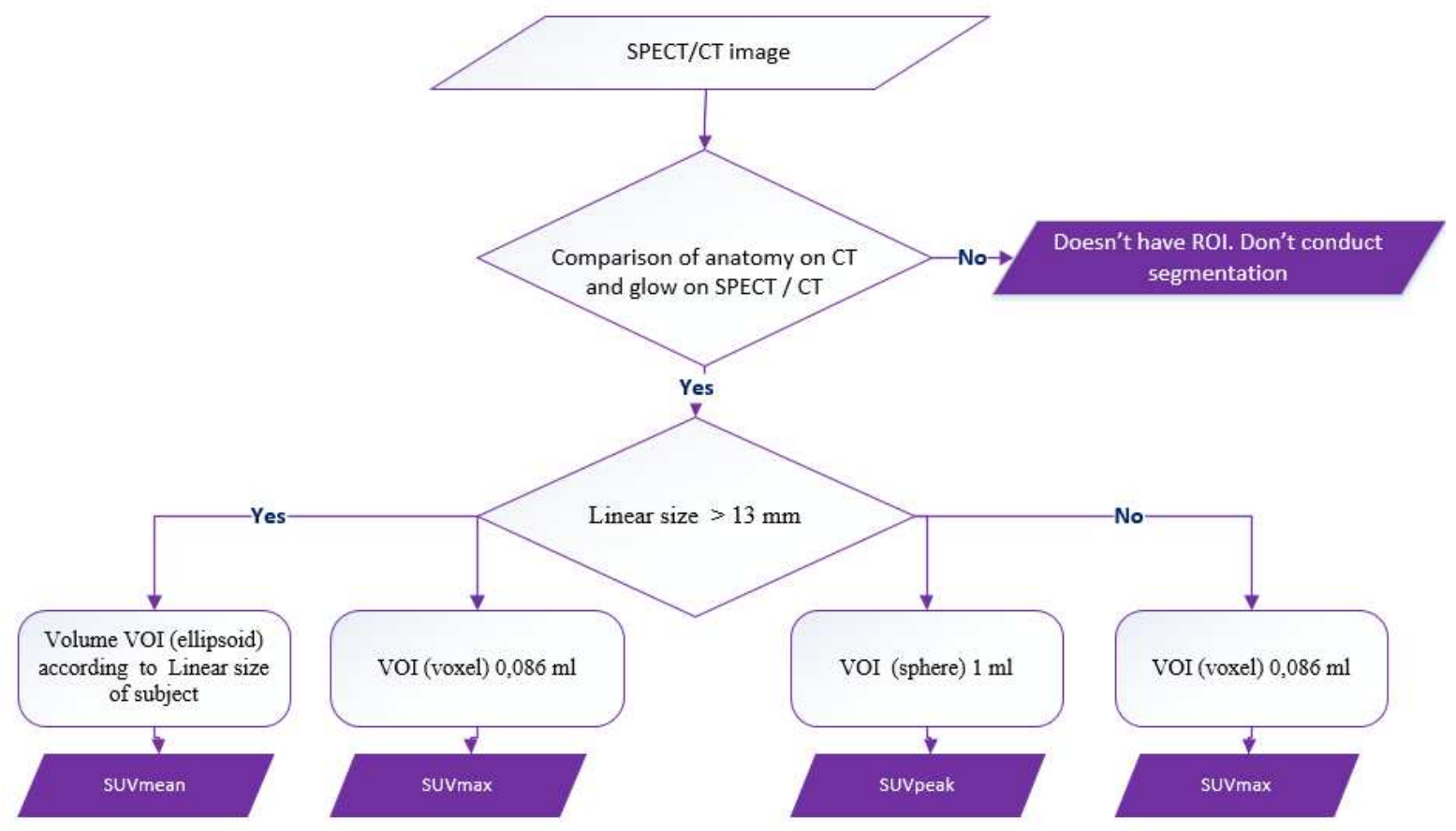

Fig 11. Algorithm segmentation

Statistical analysis was carried out for three groups dividing according to absolute risk of relapse. Descriptive statistics are put forward in table 1.

Table 1. Individual patients characteristics with DTC according to recurrence risk

\begin{tabular}{|c|c|c|c|c|c|}
\hline Characteristics & Overall & Low risk & Medium risk & High risk & P Value \\
\hline Total & $\begin{array}{c}148 \\
(100 \%)\end{array}$ & $52(32 \%)$ & $20(14 \%)$ & $76(54 \%)$ & \\
\hline \multicolumn{6}{|c|}{ Gender } \\
\hline Male & $43(29 \%)$ & $10(20 \%)$ & $6(30 \%)$ & $27(36 \%)$ & $\begin{array}{c}\mathrm{pL}-\mathrm{M}=0,354 \mathrm{pL}- \\
\mathrm{H}=0,05 \mathrm{pM}-\mathrm{H}- \\
=0,793\end{array}$ \\
\hline Female & $105(71 \%)$ & $42(80 \%)$ & $14(70 \%)$ & $49(64 \%)$ & \\
\hline Age $(95 \%, \mathrm{CI})$, years & $49,3(2,3)$ & $51,1(2,7)$ & $52,8(6,6)$ & $43,8(5,0)$ & $\begin{array}{c}\text { pL-M=0,114 pL- } \\
\mathrm{H}=0,916 \mathrm{pM}- \\
\mathrm{H}=0,108\end{array}$ \\
\hline
\end{tabular}




\begin{tabular}{|c|c|c|c|c|c|}
\hline \multicolumn{6}{|c|}{ Pathology proved } \\
\hline Yes & $144(97 \%)$ & $51(98 \%)$ & $20(100 \%)$ & $73(96 \%)$ & $\begin{array}{c}\mathrm{pL}-\mathrm{M}=0,062 \mathrm{pL}- \\
\mathrm{H}=0,006 \mathrm{pM}- \\
\mathrm{H}>0,9999\end{array}$ \\
\hline No & $4(3 \%)$ & $1(2 \%)$ & $0(0 \%)$ & $3(4 \%)$ & \\
\hline \multicolumn{6}{|c|}{ Tissue type } \\
\hline Lymph node & $48(18 \%)$ & $11(13 \%)$ & $9(19 \%)$ & $28(21 \%)$ & \multirow{2}{*}{$\begin{array}{r}\mathrm{pL}-\mathrm{M}=0,448 \\
\mathrm{pL}- \\
\mathrm{H}=0,152 \mathrm{pM}- \\
\mathrm{H}>0,9999\end{array}$} \\
\hline $\begin{array}{l}\text { Residual thyroid } \\
\text { tissue }\end{array}$ & $217(82 \%)$ & $73(87 \%)$ & $38(81 \%)$ & $106(79 \%)$ & \\
\hline $\begin{array}{l}\text { Activity (131I) } \\
\text { injection (SD), MBq }\end{array}$ & $\begin{array}{c}3988 \\
(1100)\end{array}$ & $\begin{array}{l}3223,7 \\
(728,5)\end{array}$ & $3696,2(456,4)$ & $\begin{array}{c}4589,5 \\
(1078,7)\end{array}$ & $\begin{array}{r}\mathrm{pL}-\mathrm{M}=0,0006 \\
\mathrm{pL}- \\
\mathrm{H}<0,0001 \\
\mathrm{pM}- \\
\mathrm{H}=0,002\end{array}$ \\
\hline $\mathrm{Tg}(\mathrm{SD}), \mathrm{ng} / \mathrm{ml}$ & $40,3(9,8)$ & $7,4(1,7)$ & $14,8(5,9)$ & $68,3(18,5)$ & $\begin{array}{c}\mathrm{pL}-\mathrm{M}=0,499 \mathrm{pL}- \\
\mathrm{H}=0,0143 \\
\mathrm{pM}- \\
\mathrm{H}=0,21\end{array}$ \\
\hline $\operatorname{TgAb}(\mathrm{SD}), \mathrm{IU} / \mathrm{ml}$ & $\begin{array}{l}100,8 \\
(36,4)\end{array}$ & $124,3(81,7)$ & $29,2(15,9)$ & $85,7(28,9)$ & $\begin{array}{c}\mathrm{pL}-\mathrm{M}=0,841 \mathrm{pL}- \\
\mathrm{H}=0,745 \mathrm{pM}- \\
\mathrm{H}=0,841\end{array}$ \\
\hline SUVpeak & $\begin{array}{l}117,4 \\
(11,3)\end{array}$ & $164,4(22,4)$ & $118,4(32,8)$ & $82,6(10,5)$ & $\begin{array}{c}\mathrm{pL}-\mathrm{M}=0,0690 \mathrm{pL}- \\
\mathrm{H}=0,0037 \mathrm{pM}- \\
\mathrm{H}=0,7514\end{array}$ \\
\hline SUVmax & $33,5(3,4)$ & $47,7(10,5)$ & $32(8,6)$ & $25(3,5)$ & $\begin{array}{c}\mathrm{pL}-\mathrm{M}=0,052 \mathrm{pL}- \\
\mathrm{H}=0,0033 \mathrm{pM}- \\
\mathrm{H}=0,949\end{array}$ \\
\hline
\end{tabular}

For creating predictive models, SUVpeak was used. At first the modal was designed without filtration. According to the learning group was obtained B0 $=0.046$, B $1=0.001$ - logistic regression coefficients. For patients from the test group were found value of odds relationship and probability event - repeated course of radioiodine therapy. After that, results of working the model were filled in table 2. Further, the reliability of the model is assessed depending on the threshold, by calculating its parameters based on the $2 \times 2$ table. The most appreciate parameters of the model were defined using visualization of parameter changes (Fig.12). The optimal threshold 
and set of model parameters for the logistic regression coefficients are $\mathrm{p}=0.55$ and $\mathrm{Se}=40.7 \%, \mathrm{Sp}=88.7 \%$, Accuracy $=82.2 \%, \mathrm{PPV}=41 \%, \mathrm{NPV}=88.7 \%$, respectively.

Table 2 . Test $2 \times 2$ for predictive model the second course of radioiodine therapy.

\begin{tabular}{|c|c|c|c|}
\hline \multirow{2}{*}{ Results of test } & \multicolumn{2}{|c|}{ Test $2 \times 2$} \\
\cline { 2 - 4 } & Yes repeated & No repeated & Total \\
\cline { 2 - 4 } & $\mathrm{a}=3$ & $\mathrm{~b}=10$ & $\mathrm{a}+\mathrm{b}=13$ \\
\hline Positive & $\mathrm{c}=8$ & $\mathrm{~d}=42$ & $\mathrm{c}+\mathrm{d}=50$ \\
\hline Negative & $\mathrm{a}+\mathrm{c}=11$ & $\mathrm{~b}+\mathrm{d}=52$ & $\mathrm{a}+\mathrm{b}+\mathrm{c}+\mathrm{d}=64$ \\
\hline
\end{tabular}

The second part of the investigation included a predictive model with filtration. According to the learning group was obtained $\mathrm{B}_{0}=0.473 \mathrm{~B}_{1}=0.003$ - logistic regression coefficients. The most appreciate parameters of the model were defined using visualization of parameter changes (Fig.13). The optimal threshold and set of model parameters for the logistic regression coefficients are $\mathrm{p}=0.67$ and $\mathrm{Se}=46 \%, \mathrm{Sp}=98 \%$, Accuracy $=0.89, \mathrm{PPV}=76 \%$ NPV $=87 \%$, respectively.

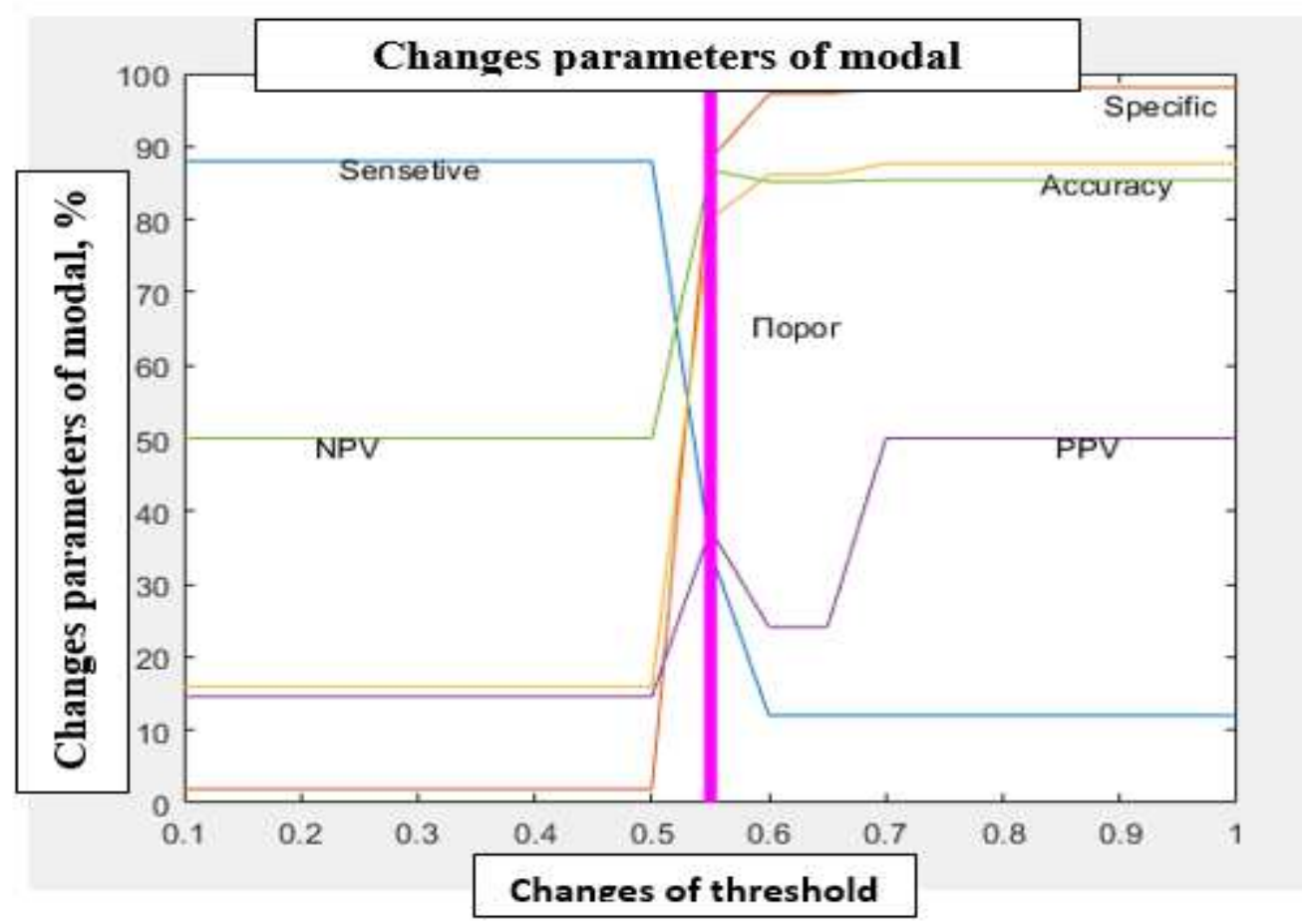

Fig 12. Changes parameters of modal without filtration. 


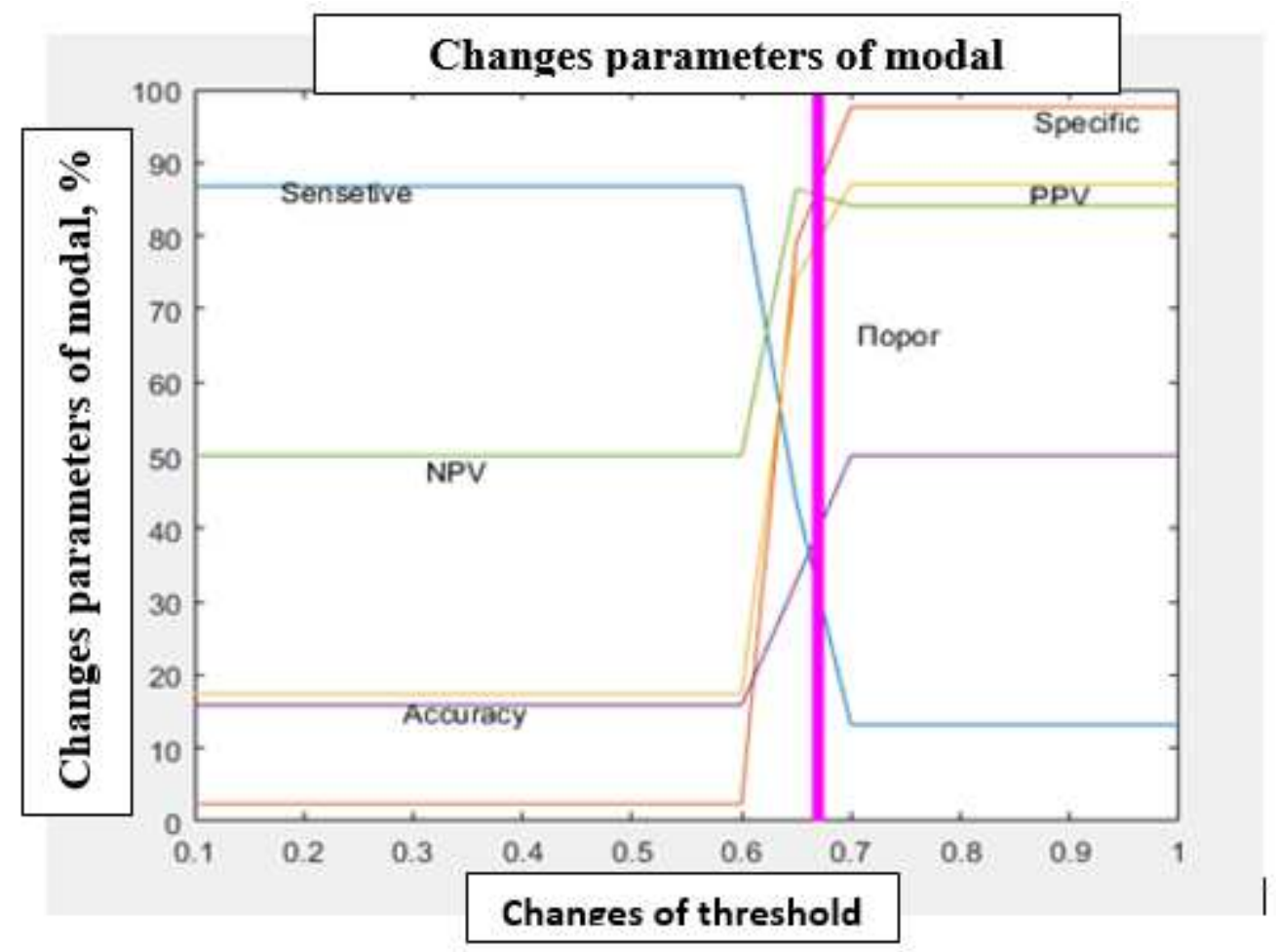

Fig 13. Changes parameters of modal with filtration.

The research showed that SUV will be able to become a part of digital patient profile in future. The main result is obtaining another tool for improving the quality of medical care.

\section{Discussion}

The calibration curve of SPECT/CT system was obtained During the first part of the investigation. However we found some characteristics which were suitable for realization of our main idea (getting an SUV for radionuclide therapy with SPECT/CT image). Majority of SPECT/CT parameters were performed by Jonathan Gear and et al. [17] The curve depends on the mean count within projection. Based on it, we made a decision that sensitivity decreases with increasing activity. The dependence is leaner. This behavior of sensitivity is a consequence of a growth in the number of decays per unit time and an overload of the spectrometric path with a large number of signals. If we didn't take into account the fact, it would not reach the universal method of determining SUV for SPECT/CT system.

The second step included investigation of spatial resolution. Work of the approach depends on spatial resolution. The value is one of the most important for our method of segmentation. The less spatial resolution of SPECT/CT, the more accuracy of SUV. Also there is an effect of partial volume for small structures. The phenomena is negative for any visualization system. There are some methods to reduce the contribution of this effect but it isn't a cheap way. [18].

During the study we distinguished exactly only 2 groups (low and high risk). According to statistical analysis, to define a medium group, it is necessary to increase the sample of patients.

As for predictive model, it is the first quantitative instrument for estimating risk of relapse DTC. Our model has some disadvantages which are a part of some innovation. Nevertheless, we have precise suggestions on how to make the model better. The main offer is to increase the number of patients in studies with a prescribed 
repeated course of radioiodine therapy. And the second part we are planning to include thyroglobulin (Tg) in the modal. It will give us the opportunity to create the first model embracing clinical and physical parameters.

\section{Conclusion}

In conclusion, this study reports the utility of SUV for SPECT/CT system. It was the first investigation which adopted SUV in radionuclide therapy but it is definitely not the least. Now we have some ideas to make our model more simple and universal for realization. There are a lot of directions to apply the new method in nuclear medicine.

\section{References}

1. Sollini M, Bandera F, Kirienko M. Quantitative imaging biomarkers in nuclear medicine: from SUV to image mining studies. Highlights from annals of nuclear medicine 2018. Eur J Nucl Med Mol Imaging. 2019;46(13):2737-2745. DOI: 10.1007/s00259-019-04531-0

2. Kinahan PE, Fletcher JW. Positron emission tomography-computed tomography standardized uptake values in clinical practice and assessing response to therapy. Semin Ultrasound CT MR. 2010;31(6):496505. DOI:10.1053/j.sult.2010.10.001

3. Marcus C, Whitworth PW, Surasi DS, Pai SI, Subramaniam RM. PET/CT in the management of thyroid cancers. AJR Am J Roentgenol. 2014. 202(6):1316-29. DOI: 10.2214/AJR.13.1167

4. Brahim A, Primakov S, Beuque M, Woodruff HC, Halilaj I, Wu G, Refaee T, Granzier R, Widaatalla Y, Hustinx R, Mottaghy FM, Lambin P. Radiomics for precision medicine: Current challenges, future prospects, and the proposal of a new framework. Methods. 2020:S1046-2023(20)30111-0. DOI: 10.1016/j.ymeth.2020.05.022

5. Rumiantsev PO, Trukhin AA, Sergunova KA, Sirota YaI, Makarova NM, Bubnov AA, Semenov DS, Ahmad ES. Phantoms for Nuclear Medicine. Medical Radiology and Radiation Safety. 2020;65(2):627. (In Russ.). DOI: 10.12737/1024-6177-2020-65-2-62-67

6. Müller C, Domnanich KA, Umbricht CA, Van der Meulen NP. Scandium and terbium radionuclides for radiotheranostics: current state of development towards clinical application. $\mathrm{Br} \mathrm{J}$ Radiol.2018:91;1093.

7. Wong KK, Gandhi A, Viglianti BL, Fig LM, Rubello D, Gross MD. Endocrine radionuclide scintigraphy with fusion single photon emission computed tomography/computed tomography. World J Radiol. 2016;8(6):635-655. DOI: 10.4329 / wjr.v8.i6.635

8. Method for assessing the risk of recurrent differentiated thyroid cancer after radioiodine therapy. Bubnov AA, Trukhin AA, Rumiantsev PO, Degtiarev MV, Serzhenko SS, Slashchuk KYU, Kolpakova EA, Dedov II, Mokrysheva NG, Melnichenko GA. ru) 2743275 C1. Patent Office: Russia. Year of publication: 2021 Application number: 2020128431 Date of registration: 26.08.2020 Date of publication: 16.02 .2021

9. Statistics adapted from the American Cancer Society's (ACS) publication, Cancer Facts \& Figures 2020, and the ACS website (January 2020). https://www.cancer.org/research/cancer-facts-statistics/all-cancerfacts-figures/cancer-facts-figures-2020.html 
10. Zubair B, Virginia AL, Fifty years of thyroid pathology: concepts and developments. Human Pathology. 2020;95:46-54, DOI: https://doi.org/10.1016/j.humpath.2019.09.008.

11. Lee R, So Y, Song YS, Lee WW. Evaluation of Hot Nodules of Thyroid Gland Using Tc-99m Pertechnetate: a Novel Approach Using Quantitative Single-Photon Emission Computed Tomography/Computed Tomography. J. Nucl. Med. Mol. 2018; 52:468-472. DOI: 10.1007/ s13139018-0554-5

12. Brady SL, Shulkin BL. Analysis of quantitative [I-123] mIBG SPECT/CT in a phantom and in patients with neuroblastoma. EJNMMI Phys 6, 31 (2019). DOI: https://doi.org/10.1186/s40658-019-0267-6

13. Simone Krebs, Joseph A. O’Donoghue, Evan Biegel, Bradley J. Beattie, Diane Reidy, Serge K. Lyashchenko, Jason S. Lewis, Lisa Bodei, Wolfgang A. Weber, Neeta Pandit-Taskar. Comparison of 68Ga-DOTA-JR11 PET/CT with dosimetric 177Lu-satoreotide tetraxetan (177Lu-DOTA-JR11) SPECT/CT in patients with metastatic neuroendocrine tumors undergoing peptide receptor radionuclide therapy. European Journal of Nuclear Medicine and Molecular Imaging (2020). https://doi.org/10.1007/s00259-020-04832-9

14. Poppe K, Bisschop P, Fugazzola L, Minziori G, Unuane D, Weghofer A: 2021 European Thyroid Association Guideline on Thyroid Disorders prior to and during Assisted Reproduction. Eur Thyroid J 2020;9:281-295. DOI: 10.1159/000512790

15. Alzen, J.L., Langdon, L.S. \& Otero, V.K. A logistic regression investigation of the relationship between the Learning Assistant model and failure rates in introductory STEM courses. IJ STEM Ed 5, 56 (2018). https://doi.org/10.1186/s40594-018-0152-1

16. Hajian-Tilaki K. Receiver Operating Characteristic (ROC) Curve Analysis for Medical Diagnostic Test Evaluation. Caspian J Intern Med. 2013;4(2):627-635.

17. Gear J, Chiesa C, Lassmann M, et al. EANM Dosimetry Committee series on standard operational procedures for internal dosimetry for 131I mIBG treatment of neuroendocrine tumours. EJNMMI Phys. 2020;7(1):15. DOI:10.1186/s40658-020-0282-7

18. Chung Ch, Hui L, Grobshtein Y, Stacy MR, Albert JS, Chi L. Noise suppressed partial volume correction for cardiac SPECT/CT. Med Phys. 2016 Sep;43(9):5225. DOI: 10.1118/1.4961391 